\title{
Caracterização da pesca artesanal com rede de camboa na Reserva Extrativista de Cassurubá, Bahia
}

\author{
Vinícius José Giglio ${ }^{1}$ \\ Matheus Oliveira Freitas ${ }^{2,3 *}$ \\ ${ }^{1}$ Universidade Estadual de Santa Cruz \\ Programa de Pós-Graduação em Ecologia e Conservação da Biodiversidade, Ilhéus - BA, Brasil \\ ${ }^{2}$ Universidade Federal do Paraná, Programa de Pós Graduação em Ecologia e Conservação \\ Caixa Postal 19031, CEP 81531-980, Curitiba - PR, Brasil \\ ${ }^{3}$ Instituto Meros do Brasil \\ * Autor para correspondência \\ serranidae@gmail.com
}

Submetido em 10/04/2012

Aceito para publicação em 17/01/2013

\section{Resumo}

Considerados relevantes áreas de berçário para peixes, os sistemas estuarinos abrigam muitas espécies comercialmente importantes. De outubro de 2008 a novembro de 2009 foi investigada a composição ictiofaunística e descrita a rotina da pesca artesanal com rede de camboa no estuário da Reserva Extrativista (RESEX) de Cassurubá, Bahia. As amostragens foram realizadas em três rios, nas luas nova e cheia. Um total de 1.074 peixes foi capturado em 17 despescas, pertencentes a 26 espécies e 17 famílias, com biomassa total de 378,4 kg. As famílias Gerreidae e Centropomidae foram as mais abundantes, dominando as capturas. Do total, $50 \%$ dos indivíduos que se encontram na primeira maturação gonadal $\left(\mathrm{L}_{50}\right)$ descrita na literatura apresentaram tamanho inferior ao esperado, indicando sobrepesca de crescimento. Recomenda-se o estabelecimento de sistemas de gestão compartilhada entre os atores envolvidos com essa arte de pesca e os gestores da Resex de Cassurubá, com o objetivo de reduzir a captura de indivíduos abaixo do tamanho de primeira maturação gonadal.

Palavras-chave: Banco dos Abrolhos; Gestão; Ictiofauna estuarina; Manguezal

\section{Abstract}

Characterization of artisanal fishery with camboa net at the Cassurubá Extractive Reserve, Bahia, Brazil. Regarded as relevant nursery areas for fishes, the estuarine systems shelter many commercially important species. From October 2008 to November 2009, we investigated the ichthyofaunal composition and described the routine of artisanal fishing with camboa net in the estuary at the Cassurubá Extractive Reserve, Bahia, Brazil. Samplings were conducted in three rivers, during the new and full moons. A total of 1,074 fishes were caught in 17 fisheries with nets, belonging to 26 species and 17 families, with a total biomass of $378.4 \mathrm{~kg}$. The Gerreidae and Centropomidae families were the most abundant ones, and there was a predominance of catches. Out of the total, $50 \%$ of individuals which were at the first gonadal maturation $\left(\mathrm{L}_{50}\right)$ described in the literature had a size less than the expected one, indicating growth overfishing. We recommends the establishment of management systems shared between the actors involved in this fishing art and the Cassurubá Extractive Reserve managers, in order to reduce the capture of individuals below the first gonadal maturation size.

Key words: Abrolhos bank reef; Estuarine ichthyofauna; Mangrove; Management 


\section{Introdução}

Criada em 2009, a Reserva Extrativista (RESEX) de Cassurubá está localizada no Extremo Sul da Bahia, em um grande complexo estuarino que abrange os municípios de Caravelas e Nova Viçosa. Abriga ecossistemas marinhos, manguezais, restingas e fragmentos de Mata Atlântica em uma área de 100.700 ha. Sua criação se deu em meio a um contexto turbulento, através da mobilização de atores e comunidades locais. A proposição da criação foi apoiada pela sociedade civil organizada, contrapondo-se a um grande projeto de carcinicultura no local em 2007. Uma das consequências deste conflito foi o fortalecimento das lideranças locais e a criação da RESEX de Cassurubá. Esta tem como objetivo principal conciliar o uso sustentável dos recursos naturais com a manutenção do meio de vida das populações tradicionais (BRASIL, 2000). A pesca na RESEX de Cassurubá é caracterizada como de subsistência e artesanal multiespecífica e representa uma importante atividade econômica para as comunidades locais (RANAURO, 2004).

A pesca com rede de camboa é tradicional em todo o norte e nordeste do Brasil e também no extremo sul da Bahia. Consiste em uma rede de malha pequena que é colocada na borda dos manguezais, cercando os peixes que estão nas raízes da vegetação durante a maré cheia. A despesca ocorre na maré baixa. As espécies de peixes mais importantes comercialmente nos estuários do nordeste do Brasil são representadas pelas famílias Ariidae, Centropomidae, Gerreidae, Mugilidae, Lutjanidae e Scianidae (CEPENE, 1999).

A captura não seletiva desta arte de pesca e as observações de desembarques com alta abundância de peixes recifais na fase juvenil poderiam estar contribuindo para a redução na abundância dos recursos pesqueiros na região. Os estuários e manguezais são alvos de impactos antrópicos, como a pesca excessiva que se destaca como causa central da perda da produtividade (SILVA, 2006). Somada a perda de habitat, as comunidades pesqueiras aceleram o processo com o uso inadequado de técnicas e petrechos de pesca que dizimam o recurso explorado (SILVA, 2006).

Hábitats costeiros como manguezais e estuários são reconhecidos como importantes berçários
(KOENIG; COLEMAN, 1998; NAGELKERKEN et al., 2001), corredores de migração e áreas de refúgio (NAGELKERKEN; VELDE, 2004; BARLETTA; BLABER, 2007) para uma variedade de espécies de peixes em diferentes estágios de vida. Este importante papel dos sistemas estuarinos também foi relatado para os manguezais do estado da Bahia, onde altas densidades de juvenis de espécies de peixes recifais comercialmente importantes foram relatadas (REIS-FILHO et al., 2010; MOURA et al., 2011).

$\mathrm{Na}$ zona costeira adjacente, está localizado o complexo recifal dos Abrolhos, o maior do Oceano Atlântico Sul Ocidental, com 60.000 km² e detentor da maior biodiversidade do Atlântico Sul (LEÃO, 1996). Alguns estudos resultaram em contribuições para subsidiar a gestão dos recursos naturais na região (ex. SANTOS et al., 2008; SCHMIDT et al., 2008; RABELO et al., 2009; FREITAS et al., 2011; MOURA et al., 2011). A pesca com camboa é uma prática ilegal, regulamentada pela Portaria SUDEPE no 466 de 1972 e, desta forma, difícil de ser monitorada. Recentemente, uma iniciativa por parte do Ministério da Pesca e Aquicultura foi implementada com o objetivo de quantificar a produção pesqueira e a sustentabilidade da pesca na região, mas não houve continuidade após o primeiro ano (V. J. Giglio, comunicação pessoal). Neste contexto, e levando em consideração a relevância socioeconômica e ecológica da região e das espécies exploradas, o presente trabalho teve como objetivo: (1) descrever a composição das capturas da pesca artesanal de rede de camboa e o contexto socioeconômico da atividade e (2) discutir e propor medidas para a gestão da pesca de camboa na RESEX de Cassurubá.

\section{Material e Métodos}

\section{Área de estudo}

A região apresenta precipitação média sem padrão claro de sazonalidade, embora os meses de maio a setembro sejam os menos chuvosos (mínima de $65 \mathrm{~mm}$ ), e os meses de outubro a janeiro os mais chuvosos (máximo $176 \mathrm{~mm}$ ) (SOARES, 2006). O estuário comporta-se como um canal de maré, governado 
por águas costeiras. $\mathrm{O}$ rio Caravelas e seus afluentes são responsáveis pelo maior aporte de água doce. A amplitude média da maré varia entre $1,10 \mathrm{~m}$ durante a quadratura e 2,9 m na sizígia (SOARES, 2006).

\section{Coleta de dados}

As amostragens foram realizadas no âmbito das atividades do Projeto Meros do Brasil, no qual foram realizadas investigações sobre a bioecologia do mero

Epinephelus itajara. Foram acompanhadas cerca de $70 \%$ das despescas de um pescador local entre outubro de 2008 e novembro de 2009. Os pesqueiros estavam localizados na região superior do estuário (áreas mais distantes da desembocadura) nos Rios da Jaburuna, do Cupido e Massangano (Figura 1). Durante o período amostral, existiam cerca de 15 redes de camboa em operação na RESEX de Cassurubá, com até $2 \mathrm{~km}$ de comprimento. A escolha da data e locais das amostragens foi feita pelo pescador, que atuou em sua rotina normal da atividade. Esta arte de pesca é influenciada pela lua, fatores ambientais e conciliada com atividades de cultivo de roça e mariscagem. A rede utilizada possuía $400 \mathrm{~m}$ de comprimento, $3 \mathrm{~m}$ de altura e malhas de $40 \mathrm{~mm}$ entre nós adjacentes no pano.

As despescas ocorreram nas marés de sizígia no período noturno (entre 22 h $30 \mathrm{~min}$ e 1 h $30 \mathrm{~min}$ ) e matutino (entre 7 h 00 min e 8 h $45 \mathrm{~min}$ ). No local, todos os peixes coletados foram identificados ao menor nível taxonômico possível. Foram utilizados como referência os manuais de Figueiredo e Menezes (1978; 1980; 2000) e Carvalho-Filho (1999) (com exceção dos representantes da família Gerreidae que foram classificados ao nível de família, devido às dificuldades de identificação em campo). Todos os indivíduos capturados foram medidos (comprimento total) e pesados em uma balança com precisão de $1 \mathrm{~g}$. Em seguida os peixes foram acondicionados em gelo e comercializados em peixarias locais ou utilizados para consumo próprio pelo pescador.

As espécies foram classificadas em quatro grupos funcionais em relação ao uso do sistema estuarino proposto por Elliot et al. (2007), sendo elas: 1) Visitantes Marinhos (VM), espécies que desovam no mar e tipicamente entram nos estuários em pequenos números; 2) Migrantes Marinhos (MM), espécies que desovam no mar e entram no estuário em grandes números, especialmente quando juvenis; 3) Estuarinos Residentes (ER), espécies estuarinas capazes de completar seu ciclo de vida dentro do ambiente; 4) Migrantes Estuarinos (ME), espécies estuarinas que têm estágios larvais de seu ciclo de vida cumpridos fora do estuário ou também são representados por populações marinhas ou dulcícolas. A classificação de acordo com o uso do habitat foi

FIGURA 1: Localização da RESEX de Cassurubá, indicando os pontos amostrados. Os círculos representam coletas realizadas em 2008 e os quadrados em 2009.

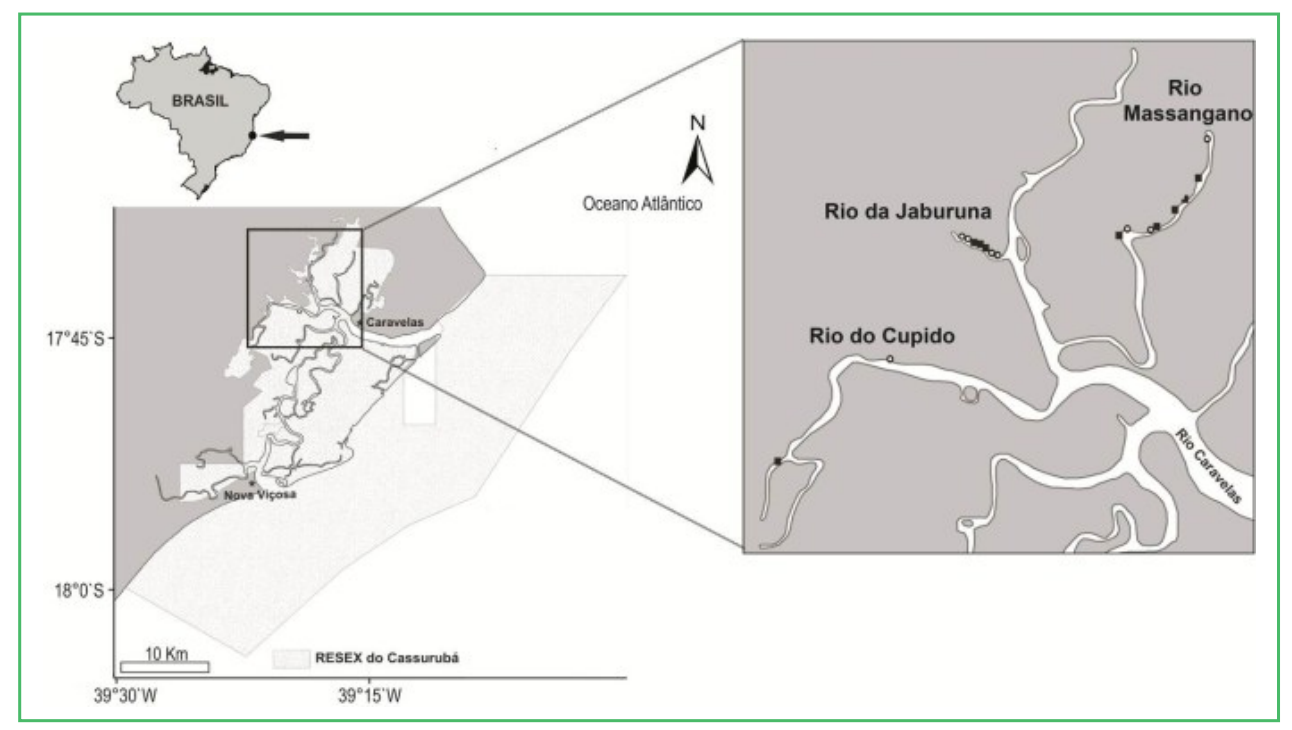


comparada com as espécies listadas em Barletta-Bergan (1999), Barletta et al. $(2003 ; 2005 ; 2008)$ e Reis-Filho et al. (2010). Os estágios de vida foram classificados em adulto e juvenil, de acordo com o tamanho mínimo de primeira maturação gonadal $\left(\mathrm{L}_{50}\right)$ disponível na literatura.

\section{Análise dos dados}

Para obter os dados da Captura por Unidade de Esforço (CPUE), foram calculadas para cada viagem de pesca: $\mathrm{CPUE}=\mathrm{C} / \mathrm{f}$, na qual, $\mathrm{C}$ é a captura em quilogramas; fé o esforço (horas que a rede permaneceu pescando). $\mathrm{O}$ esforço total por despesca foi obtido através da seguinte equação: $\mathrm{f}=$ área $\mathrm{em}^{2}$ da rede * número total de horas efetivas de pesca, obtida através do acompanhamento da instalação, armação e despesca do petrecho. As diferenças das CPUEs entre as estações do ano foram testadas através da ANOVA não paramétrica (Kruskal-Wallis). Onde os dados foram normais (verificado através do teste de normalidade de Shapiro-Wilk) a ANOVA de um fator foi usada para testar as diferenças entre as CPUEs do período matutino e noturno. Todos os testes foram realizados a um nível de significância de 0,05 .

\section{Resultados}

Ao total foram amostradas 17 despescas no rio Massangano $(n=8)$, rio da Jaburuna $(n=7)$ e rio do Cupido $(\mathrm{n}=2)$. Quatro ocorreram no período diurno e treze no noturno. As amostragens foram realizadas nas estações do inverno $(n=5)$, outono $(n=7)$ e primavera $(\mathrm{n}=4)$. No verão ocorreu apenas uma amostragem. Foram capturados 1.074 peixes de 17 famílias e 26 espécies com biomassa total de 378,4 kg (Tabela 1). A família Lutjanidae foi a mais representativa com quatro espécies, seguida por Centropomidae e Carangidae com três cada. Dez famílias estiveram representadas por uma única espécie.

A maior contribuição em número de indivíduos foi da família Gerreidae ( $\mathrm{n}=350$ e $32,59 \%)$ e biomassa (96,1 kg e 25,4\%), seguida por Centropomidae com 262 indivíduos $(24,4 \%)$ e biomassa de 102,6 $\mathrm{kg}(27,12 \%)$.
Dentre as espécies, Centropomus pectinatus (17,9\% dos indivíduos capturados) foi a mais abundante. Predominaram em ordem decrescente as espécies Chaetodipterus faber com 69 e Lutjanus alexandrei com 68 indivíduos, representando $12,75 \%$ do total. Mugil curema e Stellifer rastrifer obtiveram 60 indivíduos coletados $(11,2 \%)$ e Centropomus undecimallis 58 $(5,4 \%)$. Treze espécies contribuíram cada uma com menos de $1 \%$ da captura total em número de indivíduos (Tabela 1).

Dezenove espécies estiveram presentes nas amostragens matutinas e noturnas. Oito ocorreram somente no período noturno, todas representadas por um número reduzido de indivíduos. Com relação a utilização do habitat, a categoria Migrantes Marinhos foi a que apresentou o maior numero de espécies $(n=12)$, seguida por Estuarinos Residentes ( $\mathrm{n}=6)$, Visitantes Marinhos $(n=4)$ e Migrantes Estuarinos $(n=2)$ (Tabela 1).

$\mathrm{Na}$ distribuição em classes de tamanho, a percentagem de indivíduos abaixo do tamanho de primeira maturação gonadal variou independentemente do fato das espécies serem visitantes ou residentes. Nove espécies obtiveram capturas acima de $60 \%$ dos indivíduos abaixo do $\mathrm{L}_{50}$ (Tabela 1). De uma maneira geral, 50\% dos indivíduos que possuíam tamanho de primeira maturação gonadal descrito eram juvenis. Dentre as espécies de interesse comercial mais capturadas, $C$. undecimallis e Caranx hippos apresentaram quase a totalidade dos indivíduos capturados na fase juvenil e para as espécies L. jocu e E. itajara, todos os exemplares registrados estavam abaixo do $\mathrm{L}_{50}$, o que demonstra uma alta susceptibilidade a sobrepesca de crescimento (Figura 2). 
TABELA 1: Lista das espécies de peixes capturadas no estuário da RESEX de Cassurubá, Bahia, Brasil. N = Número total de indivíduos; $(\%)=$ Abundância relativa; $\mathrm{VM}=$ Visitantes Marinhos; ER = Estuarinos residentes; $\mathrm{MM}=$ Migrantes Marinhos; ME $=$ Migrantes Estuarinos.

\begin{tabular}{|c|c|c|c|c|c|c|c|c|c|c|c|c|}
\hline \multirow[t]{2}{*}{ Família } & \multirow[t]{2}{*}{ Espécie } & \multirow[t]{2}{*}{$\mathbf{N}$} & \multirow[t]{2}{*}{$\%$} & \multirow[t]{2}{*}{$\begin{array}{l}\text { Uso do } \\
\text { hábitat }\end{array}$} & \multicolumn{2}{|c|}{$\begin{array}{c}\text { Captura } \\
\text { por } \\
\text { período } \\
\end{array}$} & \multirow[t]{2}{*}{$\begin{array}{r}\text { Peso } \\
\text { - total }\end{array}$} & \multirow[t]{2}{*}{$\%$} & \multirow{2}{*}{$\begin{array}{l}\text { Amplitude de } \\
\text { tamanho em mm } \\
\text { (média } \pm \text { dp) }\end{array}$} & \multirow[t]{2}{*}{$\mathrm{L}_{50} \mathrm{~mm}$} & \multirow{2}{*}{$\begin{array}{c}\text { Abaixo } \\
\text { do L50 } \\
(\%)\end{array}$} & \multirow[t]{2}{*}{ Fonte } \\
\hline & & & & & Dia & Noite & & & & & & \\
\hline Ariidae & Bagre marinus & 4 & 0,37 & $\mathrm{ER}^{4}$ & 2 & 2 & 1,06 & 0,28 & $236-355(293 \pm 52,6)$ & 331 (fềmea) & 75 & Pinheiro et al. (2006) \\
\hline \multirow[t]{3}{*}{ Carangidae } & Caranx hippos & 27 & 2,51 & $\mathrm{VM}^{2}$ & 5 & 22 & 5,8 & 1,53 & $186-335(239,5 \pm 47,1)$ & $\begin{array}{l}295 \text { (fềmea) } \\
305 \text { (macho) }\end{array}$ & 81 & $\begin{array}{l}\text { Figuerola-Fernández } \\
\text { et al. (2008) }\end{array}$ \\
\hline & Selene vomer & 46 & 4,28 & $\mathrm{VM}^{4}$ & 14 & 32 & 8,6 & 2,27 & $120-350(211,4 \pm 61,2)$ & - & - & - \\
\hline & Trachinotus carolinus & 13 & 1,21 & $\mathrm{VM}^{2}$ & 5 & 8 & 3 & 0,79 & $170-260(220,8 \pm 93,9)$ & $\begin{array}{l}250 \text { (fêmea) } \\
230 \text { (macho) }\end{array}$ & 61 & $\begin{array}{l}\text { Froese e Pauly } \\
\text { (2012) }\end{array}$ \\
\hline \multirow[t]{3}{*}{ Centropomidae } & $\begin{array}{l}\text { Centropomus } \\
\text { parallelus }\end{array}$ & 12 & 1,12 & $\mathrm{ER}^{1}$ & 5 & 7 & 3,9 & 1,03 & $175-408(335,7 \pm 65,3)$ & 280 & 8 & Rodrigues (2005) \\
\hline & $\begin{array}{l}\text { Centropomus } \\
\text { pectinatus }\end{array}$ & 192 & 17,88 & $\mathrm{ER}^{1}$ & 81 & 111 & 65,8 & 17,39 & $212-540(290 \pm 91,4)$ & - & - & - \\
\hline & $\begin{array}{l}\text { Centropomus } \\
\text { undecimalis }\end{array}$ & 58 & 5,40 & $\mathrm{ER}^{1}$ & 16 & 42 & 32,9 & 8,70 & $190-810(351,1 \pm 123)$ & 680 & 96 & $\begin{array}{l}\text { Perera-García et al. } \\
\qquad(2008)\end{array}$ \\
\hline Ephippidae & Chaetodipterus faber & 69 & 6,42 & $\mathrm{MM}^{4}$ & 10 & 59 & 42,5 & 11,23 & $110-445(249,9 \pm 76,3)$ & 120 & 2 & $\begin{array}{l}\text { Froese e Pauly } \\
\text { (2012) }\end{array}$ \\
\hline Clupeidae & $\begin{array}{l}\text { Chirocentrodon } \\
\text { bleekerianus }\end{array}$ & 1 & 0,09 & $\mathrm{VM}^{2}$ & 0 & 1 & 0,1 & 0,03 & 250 & 76 (fêmea) & 0 & Corrêa et al. (2005) \\
\hline Dasyatidae & Dasyatis sp. & 1 & 0,09 & - & 0 & 1 & 3,5 & 0,93 & 450 & - & - & - \\
\hline Elopidae & Elops saurus & 3 & 0,28 & $\mathrm{MM}^{2}$ & 0 & 3 & 1,8 & 0,48 & $450-475(462,5 \pm 14,4)$ & - & - & - \\
\hline Serranidae & Epinephelus itajara* & 8 & 0,74 & $\mathrm{MM}^{4}$ & 2 & 6 & 10,7 & 2,83 & $261-645(394 \pm 117)$ & $1000-1200$ & 100 & Bullock et al. (1992) \\
\hline Gerreidae & Gerreidae & 350 & 32,59 & - & 96 & 254 & 96,1 & 25,40 & $110-460(246,4 \pm 63)$ & - & - & \\
\hline Muraenidae & Gymnothorax funebris & 3 & 0,28 & $\mathrm{MM}^{2}$ & 1 & 2 & 3,9 & 1,03 & $815-1020(901 \pm 107)$ & - & - & - \\
\hline \multirow[t]{2}{*}{ Tetraodontidae } & $\begin{array}{l}\text { Lagocephalus } \\
\text { laevigatus }\end{array}$ & 7 & 0,65 & $\mathrm{MM}^{2}$ & 0 & 7 & 2,8 & 0,74 & $210-270(235,7 \pm 22,1)$ & - & - & - \\
\hline & Sphoeroides spengleri & 18 & 1,68 & $\mathrm{MM}^{2}$ & 7 & 11 & 5,1 & 1,35 & $220-286(251 \pm 92,16)$ & - & - & - \\
\hline \multirow[t]{4}{*}{ Lutjanidae } & Lutjanus alexandrei & 68 & 6,33 & $\mathrm{MM}^{5}$ & 11 & 57 & 22,9 & 6,05 & $160-354(248,2 \pm 28)$ & $175 \dagger$ & 5 & $\begin{array}{l}\text { Fernandes et al., } \\
\text { (2012) }\end{array}$ \\
\hline & Lutjanus analis* & 3 & 0,28 & $\mathrm{MM}^{2}$ & 0 & 3 & 0,67 & 0,18 & $180-250(215 \pm 35)$ & $380-400 \dagger$ & 100 & Freitas et al. (2011) \\
\hline & Lutjanus cyanopterus * & 5 & 0,47 & $\mathrm{MM}^{2}$ & 2 & 3 & 6,42 & 1,70 & $370-550(448,2 \pm 73,5)$ & - & & - \\
\hline & Lutjanus jocu & 37 & 3,45 & $\mathrm{MM}^{5}$ & 22 & 15 & 8,96 & 2,37 & $190-322(272,4 \pm 38,7)$ & $\begin{array}{c}324 \text { (fềmea) } \\
344 \text { (macho) } \dagger\end{array}$ & 100 & Freitas et al. (2011) \\
\hline Megalopidae & Megalops atlanticus & 2 & 0,19 & $\mathrm{MM}^{4}$ & 0 & 2 & 1,42 & 0,38 & 405-419 $(412 \pm 9,9)$ & $\begin{array}{l}1250 \text { (fêmea) } \\
950 \text { (macho) }\end{array}$ & 100 & $\begin{array}{c}\text { Menezes e Paiva } \\
\text { (1966) }\end{array}$ \\
\hline \multirow[t]{2}{*}{ Mugilidae } & Mugil curema & 60 & 5,59 & $\mathrm{ME}^{2}$ & 6 & 54 & 22,61 & 5,98 & $210-415(330 \pm 39,7)$ & 275 & 10 & $\begin{array}{l}\text { Aguirre e Gallardo- } \\
\text { Cabello (2004) }\end{array}$ \\
\hline & Mugil liza & 20 & 1,86 & $\mathrm{ME}^{2}$ & 1 & 19 & 15,9 & 4,20 & $276-604(465,5 \pm 84)$ & 350 (fêmea) & 5 & $\begin{array}{l}\text { Albieri e Araújo } \\
\text { (2010) }\end{array}$ \\
\hline Pleuronectidae & Oncopterus darwini & 1 & 0,09 & - & 0 & 1 & 0,25 & 0,07 & 340 & - & - & \\
\hline Sphyraenidae & Sphyraena barracuda & 1 & 0,09 & $\mathrm{MM}^{4}$ & 0 & 1 & 1 & 0,26 & 540 & $\begin{array}{c}\text { 626-806 } \\
\text { (macho) } \\
710-985 \\
\text { (fêmea) }\end{array}$ & 100 & Kadison et al. (2010) \\
\hline \multirow[t]{3}{*}{ Sciaenidae } & Stellifer rastrifer & 60 & 5,59 & $\mathrm{ER}^{2}$ & 24 & 36 & 9,84 & 2,60 & $130-300(199,4 \pm 21,8)$ & 95 & 0 & $\begin{array}{c}\text { Camargo e Isaac } \\
\text { (2005) }\end{array}$ \\
\hline & Stellifer stellifer & 5 & 0,47 & $\mathrm{ER}^{2}$ & 1 & 4 & 0,82 & 0,22 & $175-190(182 \pm 5,7)$ & $\begin{array}{l}77 \text { (fêmea) } \\
72 \text { (macho) }\end{array}$ & 7 & $\begin{array}{l}\text { Rodrigues-Filho } \\
\text { et al. (2011) }\end{array}$ \\
\hline & Total & 1074 & 100 & & 311 & 763 & 378,4 & 100 & - & - & & \\
\hline
\end{tabular}

* Espécies listadas como ameaçadas pela União Internacional para a Conservação da Natureza: E. itajara: criticamente ameaçada; $L$. analis e L. cyanopterus: vulneravel. Fonte: ${ }^{1}$ Carpenter (2002); ${ }^{2}$ Froese e Pauly (2012); ${ }^{3}$ Figueiredo e Menezes (2000); ${ }^{4}$ Carvalho-Filho $(1999) ;{ }^{5}$ Fernandes et al. (2012). † Valores representam o comprimento padrão. 
FIGURA 2: Número de exemplares e classes de comprimento das espécies comercialmente importantes para a região. As barras escuras indicam as classes de CT abaixo da primeira maturação gonadal $\left(\mathrm{L}_{50}\right)$. C. hippos $-300 \mathrm{~mm}$ (Figuerola-Fernández et al., 2008); C. undecimalis - $680 \mathrm{~mm}$ (Perera-García et al. 2008); E. itajara - $1100 \mathrm{~mm}$ (Bullock et al.,1992); M. curema - 275 mm (Aguirre e Gallardo-Cabello, 2004); L. jocu - 324 mm (Freitas et al., 2012); C. faber - 120 mm (Froese e Pauly, 2012); L. alexandrei - $175 \mathrm{~mm}$ (Fernandes et al., 2012); S. rastrifer - $95 \mathrm{~mm}$ (Camargo e Isaac, 2005).

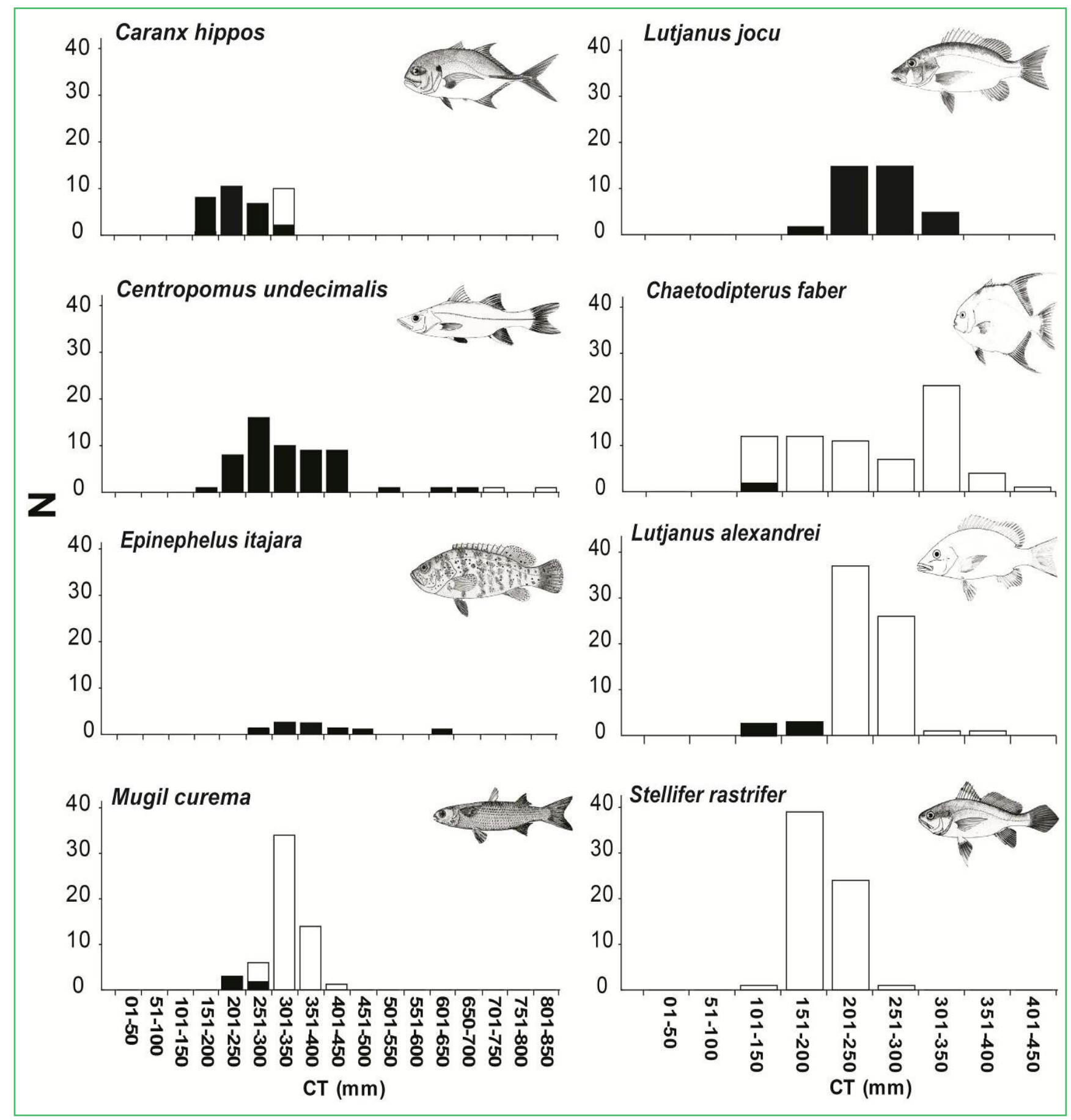


Centropomus pectinatus foi a espécie de robalo mais abundante numericamente, no entanto não existem informações sobre o tamanho de primeira maturação. O preço de venda do quilograma dos robalos (Centropomidae) obteve o valor médio de $\mathrm{R} \$ 13,00$. No entanto, a maioria dos peixes comercializados possui pequenos tamanhos e são vendidos como mistura, com o preço variando entre $R \$ 1,00$ e 3,00.

As análises da CPUE (ind. $/ \mathrm{m}^{-2}$.horas) das amostragens no período matutino e noturno não apresentaram diferenças significativas $(\mathrm{p}=0,07)$. O matutino obteve 4,3 $\pm 0,9$ indivíduos $\mathrm{m}^{-2}$ e o noturno 3,18 $\pm 1,3$ (média $\pm d p$ ). As estações do ano apresentaram CPUEs diferentes $(p<0,001)$, com maiores abundâncias nas capturas para o inverno de 2009, com média de 4,81 ind./ $\mathrm{m}^{-2}$.horas, seguido pela primavera e outono de 2009 (3,36 e 3,97 ind./ $\mathrm{m}^{-2}$.horas, respectivamente) (Figura 3).

\section{Discussão}

As capturas da pesca de camboa foram compostas por espécies de peixes estuarinos e recifais, comercialmente importantes para a região (FREITAS et al., 2011). Entretanto, o fator mais preocupante é a alta frequência de capturas de indivíduos abaixo do tamanho de primeira maturação gonadal. $\mathrm{O}$ tamanho reduzido da malha leva à captura de forma não seletiva de todo o recurso pesqueiro que é cercado pela rede. As capturas das espécies de Centropomidae, alvo desta arte de pesca, e de maior valor econômico agregado representaram $24,4 \%$ das capturas totais. No entanto, indivíduos que

FIGURA 3: Captura por unidade de esforço (CPUE) pelo número de indivíduos agrupados por estação do ano das espécies de peixes no estuário da RESEX de Cassurubá. As estações do ano correspondem a verão (janeiro a março), outono (abril a junho), inverno (julho a setembro) e primavera (outubro a dezembro). As barras indicam o desvio padrão de $0,95 \%$ e os quadrados a média. Para a estação do verão de 2009, foi realizada uma amostragem.

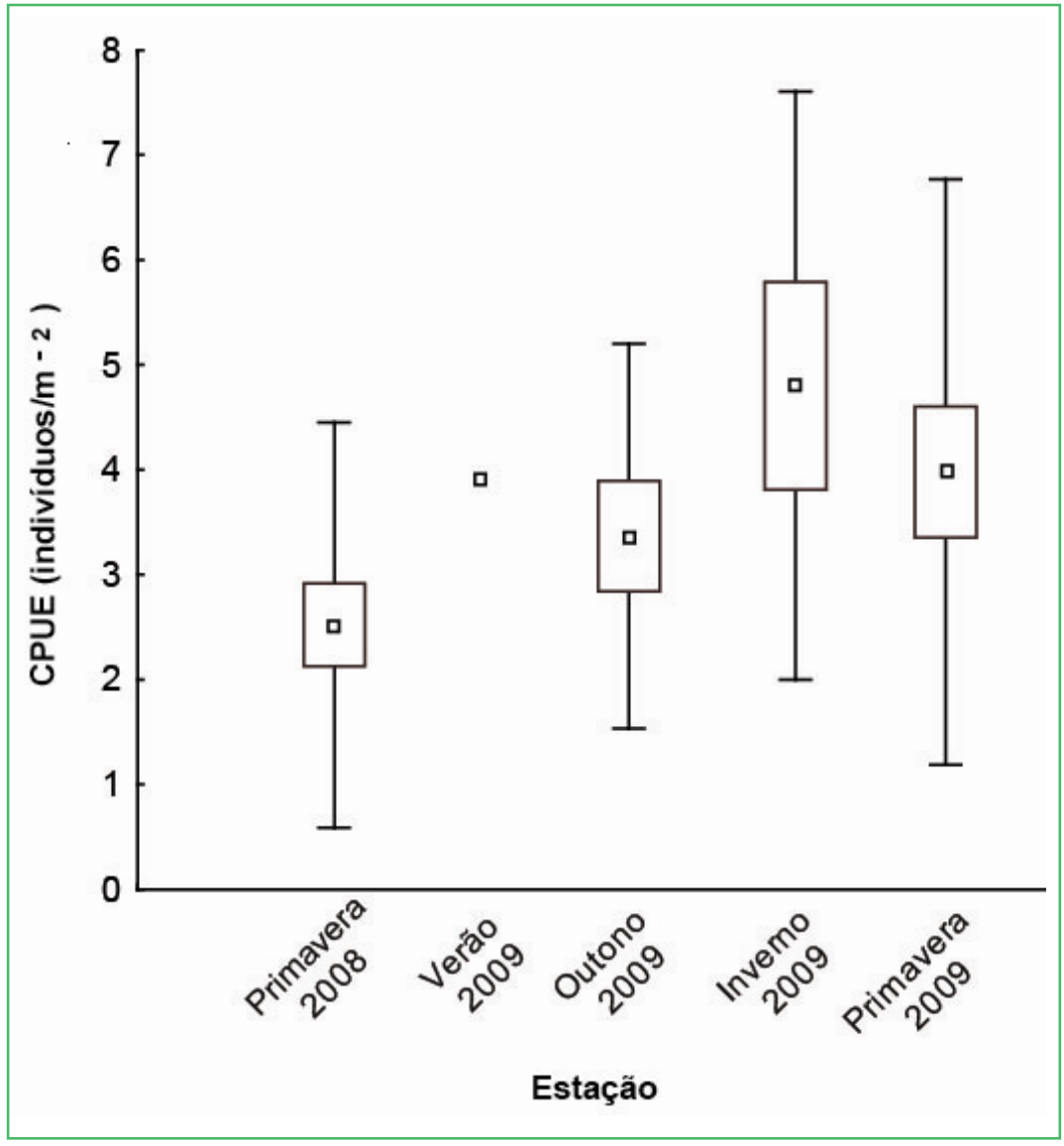


agregam maior valor para a venda estão acima dos 300 mm CT, representando apenas $39 \%$ das capturas. As espécies deste gênero possuem uma portaria específica que regulamenta um período de defeso, no entanto a pesca de camboa é praticada regularmente neste período pela maioria dos pescadores. Recente legislação no Brasil (IN-MPA/MMA 09/2012), que regulamenta a pesca amadora, incentiva que a captura seja regulada por tamanhos máximos de captura com a intenção de preservar grandes reprodutores (CHAVES, 2012). O autor ressalta ainda que a apesar da IN restringe-se à pesca amadora, se esta for bem sucedida terá potencial para expansão à pesca profissional.

A família Lutjanidae foi responsável por aproximadamente $10 \%$ do número de indivíduos e biomassa capturados. Destes, $68 \%$ estavam abaixo do tamanho de primeira maturação gonadal. A maior abundância foi verificada para L. alexandrei e L. jocu, que quando juvenis (com comprimento inferior a $10 \mathrm{~cm}$ ) habitam raízes de mangue, utilizando estas áreas como berçário (MOURA; LINDEMAN, 2007). A utilização deste habitat pelo gênero Lutjanus no nordeste brasileiro não é um fato isolado, mas um padrão ecológico de espécies desse gênero (OSÓRIO et al., 2011).

Das 26 espécies amostradas, 11 ocorrem em ambientes recifais (MOURA; FRANCINI-FILHO, 2005), o que demonstra a existência de fortes conexões entre estes ecossistemas. Estudos apontam que a ictiofauna do sistema estuarino da RESEX de Cassurubá possui conectividade com o sistema recifal do Banco dos Abrolhos, como descrito para $L$. jocu (MOURA et al., 2011). Em diversas regiões no mundo, os manguezais possuem importância direta para a manutenção da produtividade pesqueira em sistemas recifais (MUMBY et al., 2004).

A pesca artesanal de peixes recifais, principalmente das famílias Lutjanidae e Serranidae, representam uma importante fonte de renda para grande parte da população dos municípios do entorno do Banco dos Abrolhos (MARCHIORO et al., 2005; FREITAS et al., 2011). A perda de produtividade dos recursos explotados é particularmente agravada com a degradação sofrida pelos ecossistemas costeiros (LESSA et al., 2004). Estes estoques podem estar sofrendo uma pressão de pesca intensa nas populações juvenis. Isto pode comprometer as pescarias como um todo, como já ocorre para $E$. itajara que tem moratória de pesca estabelecida no Brasil desde 2002 e é classificada como criticamente ameaçada pela União Internacional para a Conservação da Natureza - IUCN. KLIPPEL et al. (2005) descrevem ainda níveis ideais de mortalidade por pesca para $L$.jocu e quadro moderado de sobreexplotação para $L$. analis, que também figura como Vulnerável pela IUCN.

As redes de camboa geralmente são colocadas em áreas com maior complexidade de hábitat (presença de Rizophora). Nestas, ocorrem as mais altas densidades de peixes juvenis (NAGELKERKEN; VELDE, 2002), que são atraídos por benefícios como abundância de alimento e proteção contra predadores (PATERSON; WHITFIELD, 2000). Estas características, somadas ao tamanho reduzido da malha, tornam a pesca com rede de camboa uma prática com relevantes impactos para as espécies capturadas. Comparações das capturas através de redes com os tamanhos de malha permitidos (acima de $70 \mathrm{~mm}$ ) são um importante tópico para futuros estudos. Apesar dos dados apresentados serem oportunistas e descritivos, acredita-se que são informações relevantes para manejo da pesca de camboa na RESEX de Cassurubá e pescarias recifais, que envolvem diversas comunidades pesqueiras ao longo do Banco dos Abrolhos.

A portaria sobre pesca em águas interiores é antiga (Portaria SUDEPE no 466 de 1972), mas os pescadores afirmam que antes da existência da RESEX de Cassurubá não possuíam ciência sobre a sua vigência. Entretanto, esta atividade é uma importante fonte de renda para cerca de 15 famílias de baixa renda. A maioria destes pescadores não tem acesso às políticas de apoio à classe pesqueira, porque eles não têm condições de arcar com despesas mensais das Colônias de Pesca, e se desencorajam perante a burocracia existente.

No atual contexto dos recursos pesqueiros no Brasil, são necessárias mudanças de paradigma sobre o processo de gestão. Na prática, inúmeros sistemas de gestão compartilhada têm sido estabelecidos como uma forma mais efetiva para o gerenciamento sustentável dos recursos pesqueiros, sendo implementados ao longo das bacias hidrográficas e da zona costeira brasileira (KALIKOSKI et al., 2009). As RESEXs surgem como 
uma ferramenta para viabilizar estes processos, pois legalmente podem estabelecer suas próprias regras de uso, desde que mais restritivas em relação a regras de instâncias superiores como a esfera federal (MOURA et al., 2009; SEIXAS et al., 2009). Na RESEX de Cassurubá, a pesca com rede de camboa, além de proibida, se mostrou insustentável ao exercer uma considerável pressão de pesca sobre a população juvenil de espécies comercialmente importantes para a região.

\section{Agradecimentos}

Ao pescador camboeiro que nos permitiu acompanhar sua rotina. A H. Teixeira e P. Beckenkamp pelo apoio nas coletas. A ECOMAR pela execução. A H.L. Spach pela revisão do manuscrito. A RESEX de Cassurubá/ICMBio pela autorização do estudo. As atividades foram financiadas pela Petrobras, por meio do Programa Petrobras Ambiental.

\section{Referências}

AGUIRRE, A. L. I.; GALLARDO-CABELLO, M. Reproduction of Mugil cephalus and M. curema (Pisces: Mugilidae) from a coastal lagoon in the Gulf of Mexico. Bulletin of Marine Science, Miami, v. 75, n. 1, p. 37-49, 2004.

ALBIERI, R.J.; ARAÚJO, F. G. Reproductive biology of the mullet Mugil liza (Teleostei: Mugilidae) in a tropical Brazilian bay. Zoologia, Curitiba, v. 3, p. 331-340, 2010.

BARLETTA, M.; AMARAL, C. S.; CORRÊA, M. F. M.; GUEBERT, F.; DANTAS, D. V.; LORENZI, L.; SAINT-PAUL, U. Factors affecting seasonal variations in demersal fish assemblages at an ecocline a tropical-subtropical estuary. Journal of Fish Biology, Hoboken, v.73, p. 1314-1336, 2008.

BARLETTA, M.; BARLETTA-BERGAN, A.; SAINT-PAUL, U.; HUBOLD, G. Seasonal changes in density, biomass, and diversity of estuarine fishes in tidal mangrove creeks of the lower Caeté estuary (northern Brazilian coast, east amazon). Marine Ecology Progress Series, Oldendorf, v. 256, p. 217-228, 2003.

BARLETTA, M.; BARLETTA-BERGAN, A.; SAINT-PAUL, U.; HUBOLD, G. The role of salinity in structuring the fish assemblages in a tropical estuary. Journal of Fish Biology, Hoboken, v. 66, p. 45-72, 2005.

BARLETTA, M.; BLABER, S. J. M. Comparison of fish assemblages and guilds in tropical habitats of the Embley (Indo West Pacific) and Caeté (Western Atlantic) estuaries. Bulletin of Marine Science, Miami, v. 80, n. 3, p. 647-680, 2007.

BARLETTA-BERGAN, A. Structure and seasonal dynamics of larval and juvenile fish in the mangrove-fringed estuary of the rio Caeté in north Brazil. Bremen: Zentrum Fur Marine Tropenokologie, Contribution 8, 1999. 220 p.
BRASIL. Lei no 9.985, de 18 de julho de 2000. Lei do Sistema Nacional de Unidades de Conservação da Natureza (SNUC). 2000. BULLOCK, L. H.; MURPHY, M. D.; GODCHARLES, M. F.; MITCHELL, M. E. Age, growth and reproduction of jewfish Epinephelus itajara in the eastern Gulf of Mexico. Fishery Bulletin, Seattle, v. 90, p. 243- 249, 1992.

CAMARGO, M.; ISAAC, V. Reproductive biology and spatiotemporal distribuition of Stellifer rastrifer, Stellifer naso and Macrodon ancylodon (Scianidae) in the Caeté Estuary, Northern Brazil. Brazilian Journal of Oceanography, São Paulo, v. 53, n. 1, p. 13-21, 2005.

CARPENTER, K. E. The living marine resources of the Western Central Atlantic. FAO Species Identification Guide for Fishery Purposes and American Society of Ichthyologists and Herpetologists, Roma, v. 2, n. 1, p. 601-1374, 2002.

CARVALHO-FILHO, A. Peixes da costa brasileira. São Paulo: Melro, 1999. 320 p.

CEPENE. Boletim estatístico da pesca marítima e estuarina no estado da Bahia - 1998. Tamandaré: Edições IBAMA. CEPENE, 1999. $32 \mathrm{p}$.

CHAVES, P. T. Tamanho de maturação como instrumento de gestão pesqueira: uma revisão crítica. Acta Biológica Paranaense, Curitiba, v. 41, n. 3-4, p. 127-132, 2012.

CORRÊA, C. E.; CHAVES, P. T.; GUIMARÃES, P. R. B. Biology of Chirocentrodon bleekerianus (Poey, 1867) (Clupeiformes: Pristigasteridae) in a Continental Shelf Region of Southern Brazil, Brazilian Archives of Biology and Technology, Curitiba, v. 48, n. 3, p. 419-427, 2005.

ELLIOTT, M.; WHITFIELD, A. K.; POTTER, I. C.; BLABER, S. J. M.; CYRUS, D. P.; NORDLIE, F. G.; HARRISON, T. D. The guild approach to categorizing estuarine fish assemblages: a global review. Fish and Fisheries, Malden, v. 8, p. 241-268, 2007.

FERNANDES, C. A. F.; OLIVEIRA, P. G. V.; TRAVASSOS, P. E. P.; HAZIN, F. V. H. Reproduction of the Brazilian snapper, Lutjanus alexandrei Moura \& Lindeman, 2007 (Perciformes: Lutjanidae), off the northern coast of Pernambuco, Brazil. Neotropical Ichthyology, Porto Alegre, v. 10, n. 3, p. 587-592, 2012.

FIGUEIREDO, J. L.; MENEZES, N. A. Manual de peixes marinhos do sudeste do Brasil. II. Teleostei (1). São Paulo: Ed. Universidade de São Paulo, 1978. 110 p

FIGUEIREDO, J. L.; MENEZES, N. A. Manual de peixes marinhos do sudeste do Brasil. VI. Teleostei (3). São Paulo: Ed. Universidade de São Paulo, 1980. 96 p.

FIGUEIREDO, J. L.; MENEZES, N. A. 2000. Manual de peixes marinhos do sudeste do Brasil. VI. Teleostei (5). São Paulo: Ed. Universidade de São Paulo, 2000. 116 p.

FIGUEROLA-FERNÁNDEZ, M.; PENA-ALVARADO, N.; TORREZ-RUIZ, W. Aspects of the reproductive biology of recreationally important fish species in Puerto Rico. Puerto Rico: US Fish ans Wildlife Service, 2008. 134 p.

FITZHUGH, G. R.; KOENIG, C. C.; COLEMAN, F. C.; GRIMES, C. B.; STURGES, W. A. Spatial and temporal patterns in fertilization and settlement of young gag (Mycteroperca microlepis) along the West Florida Shelf. Bulletin of Marine Science, Miami, v. 77, p. 377-396, 2005.

FREITAS, M. O.; MOURA, R. L.; FRANCINI-FILHO, R. B.; MINTE-VERA, C. V. Spawning patterns of commercially important 
reef fish (Lutjanidae and Serranidae) in the tropical western South Atlantic. Scientia Marina, Barcelona, n. 75, v. 1, p. 134-146, 2011. FROESE, R.; PAULY, D. (Ed.). Fishbase. 2012. Disponível em: $<$ http:www.fishbase.com>. Acesso em: 20 jan. 2012.

KADISON, E.; D’ALESSANDRO, E. K.; DAVIS, G. O.; HOOD, P. B. Age, growth and reproductive patterns of the great barracuda, Sphyraena barracuda, from the Florida Keys. Bulletin of Marine Science, Miami, v. 86, n. 4, p. 773-784, 2010.

KALIKOSKI， D. C.; SEIXAS， C. S.; ALMUDI, T. Gestão compartilhada e comunitária da pesca no Brasil: avanços e desafios. Ambiente \& Sociedade, Campinas, v. 12, n. 1, p. 151-172, 2009.

KLIPPEL, S.; OlAVO, G.; COSTA, P. A. S.; MARTINS, A. S.; PERES, M. B. Avaliação dos estoques de lutjanídeos da costa central do Brasil: Análise de coortes e modelo preditivo de Thompson e Bell para comprimentos. In: COSTA, P. A. S.; MARTINS, A. S.; OLAVO, G. (Ed.). Pesca e potenciais de exploração de recursos vivos na região central da Zona Econômica Exclusiva brasileira. Rio de Janeiro: Museu Nacional, 2005. p. 83-98.

KOENIG, C. C.; COLEMAN, F. C. Absolute abundance and survival of juvenile gags in sea grass beds of the northeastern Gulf of Mexico. Transactions of the American Fisheries Society, Oxford, v. 127, p. 44-55, 1998.

LEÃO, Z. M. A. N. The coral reefs of Bahia: morphology, distribution and the major environmental impacts. Anais da Academia Brasileira de Ciências, Rio de Janeiro, v. 3, n. 68, p. 439-452, 1996.

LESSA, R. P.; BEZERRA JR., J. L.; NÓBREGA, M. F. Programa de amostragem do SCORE-NE: análise dos desembarques. In: LESSA, R. P.; NOBREGA, M. F.; ARAUJO, B. A. M.; BEZERRA JUNIOR, J. L. (Ed.). Dinâmica das frotas pesqueiras da região nordeste do Brasil. Recife: Universidade Federal Rural de Pernambuco/DIMAR, 2004. p. 43-101.

MARCHIORO, G. B.; NUNES, N. A.; DUTRA, G. F.; MOURA, R. L.; PEREIRA, P. G. 2005. Avaliação dos impactos da exploração e produção de hidrocarbonetos no Banco dos Abrolhos e adjacências. Megadiversidade, Belo Horizonte, v. 1, n. 2, p. 225-310, 2005.

MENEZES, M. F.; PAIVA, P. Notes on the biology of tarpon, Tarpon atlanticus (Cuvier and Valenciennes), from coastal waters of Ceara State, Brazil. Arquivos da Estação de Biologia Marinha, Fortaleza, v. 6, p. 83-98, 1966.

MOURA, R. L; FRANCINI-FILHO, R. B. Reef and shore fishes of the Abrolhos Region, Brazil. In: DUTRA, G. F.; ALLEN, G. R.; WERNER, T.; MCKENNA, S. A. (Ed.). A rapid marine biodiversity assessment of the Abrolhos Bank, Brazil. Washington: Conservation International, 2005. 160 p.

MOURA, R. L.; FRANCINI-FILHO, R. B.; CHAVES, E. M.; MINTE-VERA, C. V.; LINDEMAN, K. C. Use of riverine through reef habitat systems by dog snapper (Lutjanus jocu) in Eastern Brazil. Estuarine, Coastal and Shelf Science, Amsterdam, v. 95, n. 1, p. 1-5, 2011.

MOURA, R. L.; LINDEMAN, K. C. A new species of snaper (Perciformes: Lutjanidae) from Brazil, with comments on the distribution of Lutjans griseus and L. apodus. Zootaxa, Auckland, v. 1422, p. 31-43, 2007.

MOURA, R. L; MINTE-VERA, C. V.; CURADO, I. B.; FRANCINI-FILHO, R. B.; RODRIGUES, H. C. L.; DUTRA, F. D.; ALVES, D. C. ; SOUTO, F. J. B. Challenges and prospects of fisheries co-management under a Marine Extractive Reserve framework in Northeastern Brazil. Coastal Management, London, v. 37, n. 6, p. 617-632, 2009.

MUMBY, P. J.; EDWARD, A. J.; ARIAS-GONZÁLEZ, J. E.; LINDEMAN, K. C.; BLACKWELL, P. G.; GALL, A.; GORCZYNSKA, M. I.; HARBORNE, A. R.; PESCOD, C. L.; RENKEN, H.; WABNITZ, C. C. C.; LLEWELLYN, G. 2004. Mangroves enhance the biomass of coral reef fish communities in the Caribbean. Nature, London, v. 427, p. 533-536. 2004.

NAGELKERKEN, I.; KLEIJNEN, S.; KLOP, T.; VAN DEN BRAND, R. A. C. J.; COCHERET DE LA MORINIÈRE, E.; VELDE, G. van der. Dependence of Caribbean reef fishes on mangroves and seagrass beds as nursery habitats: a comparison of fish faunas between bays with and without mangroves/seagrass beds. Marine Ecology Progress Series, Oldendorf, v. 214, p. 225 235, 2001

NAGELKERKEN, I.; VELDE, G. van der. Do non-estuarine mangroves harbour higher densities of juvenile fish than adjacent shallow-water and coral reef habitats in Curaçao (Netherlands Antilles)? Marine Ecology Progress Series, Oldendorf, v. 245, p. 191-204, 2002.

NAGELKERKEN, I.; VELDE, G. van der. Relative importance of interlinked mangroves and seagrass beds as feeding habitats for juvenile reef fish on a Caribbean island. Marine Ecology Progress Series, Oldendorf, v. 274, p. 153-159, 2004.

OSÓRIO, F. M.; GODINHO, W. O.; LOTUFO, T. M. C. 2011. Ictiofauna associada às raízes de mangue do estuário do Rio Pacoti - CE, Brasil. Biota Neotropica, Campinas, v. 11, n. 1, p. 415-420, 2011.

PATERSON, A. W.; WHITFIELD, A. K. Do shallow-water habitats function as refugia for juvenile fishes? Estuarine Coastal and Shelf Science, Amsterdam, v. 51, p. 359-364, 2000.

PERERA-GARCÍA, M.; MENDOZA-CARRANZA, M.; PÁRAMO-DELGADILLO, S. Reproductive and population dynamics of common snook Centropomus undecimalis (Perciformes: Centropomidae) in Barra San Pedro, Centla, Mexico. Universidad y Ciencia: Trópico Húmedo, Tabasco, v. 24, n. 1, p. 49-59, 2008

PINHEIRO, P.; BROADHURST, M. K.; HAZIN, F. H. V.; BEZERRA, T.; HAMILTON, S. Reproduction in Bagre marinus (Ariidae) off Pernambuco, northeastern Brazil, Journal of Applied Ichthyology, Hoboken, v. 22, p. 189-192, 2006.

RABELO, L. B.; MUTO, E. Y.; SOARES, L. S. H. Observações preliminares sobre o hábito alimentar do robalo-flecha Centropomus undecimalis (Bloch, 1792) e robalo-peba Centropomus parallelus (Poey,1860) no estuário de Caravelas (Bahia-Brasil). Boletim Técnico Científico do CEPENE, Tamandaré, v. 17, n. 1, p. 89-96, 2009.

RANAURO, M. L. 2004. Levantamento socioeconômico e cultural da área proposta para criação da Unidade de Conservação de Uso Sustentável de Cassurubá e de seu entorno. Caravelas: IBAMA/CEPENE IBAMA/DIREC/PNM dos Abrolhos/Conservação Internacional. 2004. 115 p.

REIS-FILHO, J. A.; NUNES, J. A. C.; FERREIRA, A. Estuarine ichthyofauna of the Paraguaçu river, Todos os Santos bay, Bahia, Brazil. Biota Neotropica, São Paulo, v. 10, n. 4, p. 301-311, 2010. 
RODRIGUES, P. P. Aspectos reprodutivos do robalo peba, Centropomus parallelus, na foz do Rio Doce, Linhares/ES. 2005. 51 f. Monografia (Graduação em Oceanografia) - Universidade Federal do Espírito Santo, Vitória. 2005.

RODRIGUES-FILHO, J. L.; VERANI, J. R.; PERET, A. C.; SABINSON, L. M.; BRANCO, J. O. The influence of population structure and reproductive aspects of the genus Stellifer (Oken, 1817) on the abundance of species on the southern Brazilian coast. Brazilian Journal of Biology, São Carlos, v. 71, n. 4, p. 991-1002, 2011.

SANTOS, M. C. F.; ALMEIDA, L.; SILVA, C. G. M. Avaliação quali-quantitativa da ictiofauna acompanhante na pesca do camarão sete-barbas Xiphopenaeus kroyeri (Heller,1862) no município de Caravelas (Bahia-Brasil). Boletim Técnico Científico do CEPENE, Tamandaré, v. 16, n. 1, p. 99-107, 2008.

SCHMIDT, A. J.; THEIL, C. M. I.; GALLI, O. B. S. Estudos preliminares sobre efeitos de uma mortalidade em massa em uma população de caranguejo-uçá, Ucides cordatus (Linnaeus, 1763) (Crustacea, Decapoda, Brachyura), em Caravelas (Bahia -Brasil). Boletim Técnico Científico do CEPENE, Tamandaré, v. 16, n. 1, p. 43-49, 2008.
SEIXAS, C. S.; MINTE-VERA, C. V.; FERREIRA, R. G., MOURA, R. L.; CURADO, I. B.; PEZZUTI, J.; THÉ, A. P. G.; FRANCINI-FILHO, R. B. Co-managing commons: advancing aquatic resources management in Brazil. In: LOPES, P.; BEGOSSI, A. (Ed.). Current trends in human ecology. Newcastle upon Tyne: Cambridge Scholars Publishing, 2009. p. 156-182.

SILVA, J. B. Territorialidade da pesca no estuário de ItapessocaPE: técnicas, petrechos, espécies e impactos ambientais. 2006. 86 f. Dissertação (Mestrado em Geografia) - Universidade Federal de Pernambuco, Recife. 2006.

SOARES, M. L. G. Laudo biológico do sistema Caravelas - Nova Viçosa com vistas à criação da Reserva Extrativista de Cassurubá. Rio de Janeiro: Núcleo de Estudos em Manguezais (NEMA). Departamento de Oceanografia. Universidade do Estado do Rio de Janeiro. 2006. 246 p. 